\title{
The Association between Facial Morphology and Cold Pattern
}

\author{
Ilkoo Ahn, Kwang-Ho Bae, Hee-Jeong Jin, Siwoo Lee* \\ Korean Medicine Data Division, Korea Institute of Oriental Medicine
}

\begin{abstract}
Objectives: Facial diagnosis is an important part of clinical diagnosis in traditional East Asian Medicine. In this paper, using a fully automated facial shape analysis system, we show that facial morphological features are associated with cold pattern.

Methods: The facial morphological features calculated from 68 facial landmarks included the angles, areas, and distances between the landmark points of each part of the face. Cold pattern severity was determined using a questionnaire and the cold pattern scores (CPS) were used for analysis. The association between facial features and CPS was calculated using Pearson's correlation coefficient and partial correlation coefficients.

Results: The upper chin width and the lower chin width were negatively associated with CPS. The distance from the center point to the middle jaw and the distance from the center point to the lower jaw were negatively associated with CPS. The angle of the face outline near the ear and the angle of the chin line were positively associated with CPS. The area of the upper part of the face and the area of the face except the sensory organs were negatively associated with CPS. The number of facial morphological features that exhibited a statistically significant correlation with CPS was 37 (unadjusted).

Conclusions: In this study of a Korean population, subjects with a high CPS had a more pointed chin, longer face, more angular jaw, higher eyes, and more upward corners of the mouth, and their facial sensory organs were relatively widespread.
\end{abstract}

$\overline{K e y}$ Words $:$ cold pattern, cold sensitivity, facial diagnosis, traditional Chinese medicine

\section{Introduction}

Pattern identification is the most representative component of the diagnostic process used in traditional East Asian medicine (TEAM), which distinguishes it from Western medicine. It is known that pattern identification can be helpful for understanding a patient's health status and choosing the appropriate medical treatment. Recent studies have shown that pattern identification provides practical insights for disease recognition and medical intervention, helping to improve the health of patients in both Eastern and Western medicine ${ }^{1-3)}$. Among pattern identification types, cold pattern is considered the most important scale used to survey a patient's health status because it describes the basic nature of the imbalance in the body. The symptoms of an

\footnotetext{
- Received : 22 July 2021

- Revised : 1 September 2021

- Accepted : 15 October 2021

- Correspondence to : Siwoo Lee

Korean Medicine Data Division, Korea Institute of Oriental Medicine

1672 Yuseong-daero, Yuseong-gu, Daejeon 34054, Republic of Korea

Tel : +82-42-868-9555, Fax : +82-42-861-5800, E-mail : ifree72@gmail.com
} 
individual with a cold pattern include aversion to cold temperature, cold limbs, white face, no thirst, no perspiration, copious clear urine, and diarrhea ${ }^{4)}$. According to recent studies, cold pattern is correlated with sex, body mass index $(\mathrm{BMI})^{5-7)}$, metabolic rate $^{5)}$, glucose metabolism ${ }^{6}$, the sympathetic nervous system ${ }^{6,7)}$, thyroid function $^{8)}$, the renin-angiotensin system ${ }^{9)}$, adrenal hormones ${ }^{10,11)}$, and anemia ${ }^{11)}$.

Facial diagnosis is an important diagnostic method that has been used by many practitioners in and outside of TEAM ${ }^{12)}$. The face is a good indicator of overall health and wellbeing, because it reflects the symptoms, cause, and origin of the disease. The face can be considered a mirror that reflects internal health or wellness. Many studies have investigated the use of facial images for quantitative differential diagnosis ${ }^{12)}$, including computerized facial diagnosis systems for hepatitis ${ }^{13)}$, coronary heart disease ${ }^{14)}$, and diabetes mellitus ${ }^{15}$. Recent studies have also shown that facial appearance is associated with hormone levels ${ }^{16)}$, perceived health ${ }^{16,17)}$, $\mathrm{BMI}^{18,19)}$, visceral obesity $^{20)}$, and body weight ${ }^{21)}$.

In TEAM, the facial shape has been used to diagnose the constitution rather than the cold and heat pattern based on the view that it reflects the individual characteristics ${ }^{22,23)}$. Although the cold and heat pattern also refers to the body's response to a condition, it includes the concept of individual characteristics associated with the physiological or pathological characteristics of the body, namely, constitution ${ }^{24-26)}$. Many studies on cold constitution have been conducted in Japan ${ }^{24,25)}$ and twin studies have reported the heritability of the cold and heat pattern recently in Korea ${ }^{26)}$. The constitutions of Qi deficiency, Yang deficiency, Yin deficiency, Blood deficiency are types of constitution showing the cold and heat pattern in the theory of constitution of $\mathrm{TCM}^{27)}$.

In this paper, we showed that cold pattern severity was associated with facial morphological features using a fully automated facial shape analysis system. We automatically extracted facial landmark points from facial images acquired with a digital camera. After computing the morphological features, such as distances, angles, and areas from the extracted landmark points, we investigated their association with cold pattern symptoms. To our knowledge, this study is the first attempt to objectively analyze the correlation between facial morphological features and cold pattern.

\section{Methods}

\section{Participants}

This cross-sectional study was conducted using the medical data of 452 Korean participants attending two medical institutes in Korea between October 2015 and December 2015. These data were stored at the Korean Medicine Data Center $(\mathrm{KDC})^{28)}$ (Figure 1) and there were no missing data. These subjects were previously recruited for three studies on persistent or recurrent fatigue (40 patients), neck pain (372 patients), and sleeping problems (40 patients). The inclusion and exclusion criteria for participants for each study are detailed in the Supplementary Material. The participants were between 30 and 49 years of age with BMIs ranging from 15.7 to 34.1. 


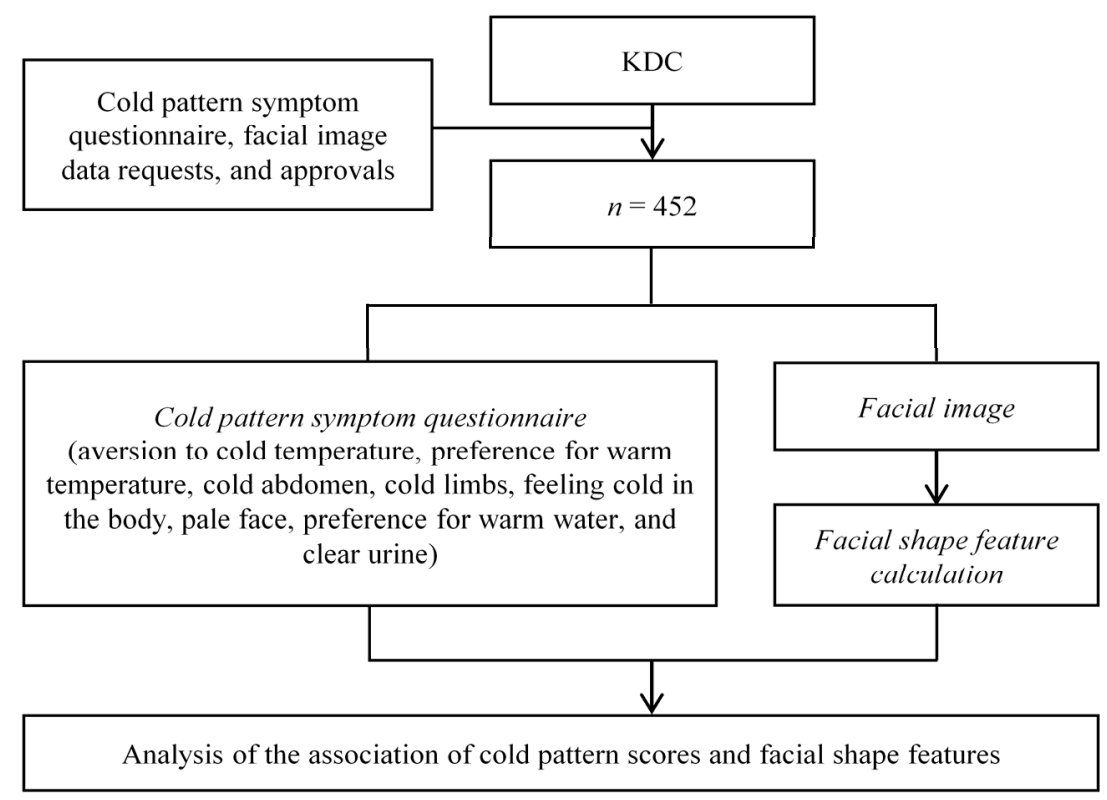

Fig. 1. Patient recruitment and study flow. KDC, Korean Medicine Data Center.

The hospital's institutional review board approved the study protocol (KHNMCIH 2014-09-010), and all participants gave written informed consent prior to their inclusion in the study.

\section{Facial morphological features}

In this subsection, we describe the computation of facial shape features from a facial image. While the shape of a face can be defined in various ways, in this paper, the facial shape features are defined as the angles, areas, and distances between the landmark points of each part of the face. The feature computation process is explained in detail in the following subsections (see Figure 2).

\section{1) Image acquisition}

The purpose of the study was explained to the participants at the beginning of the facial image acquisition process. Participants were asked to wear a hair band to prevent their hair from covering their forehead and ears. They were allowed to sit comfortably in a chair, and a camera was placed 1.6 meters away from them. Frontal face images of the participants were taken using a digital camera (Nikon D5100 with an 85-mm lens; Nikon Co., Ltd., Japan) equipped with a prime lens (a lens with only one focal length). We prepared a strict standard operating procedure for photo-taking to reduce inter-rater bias. All facial images were taken at the same location, with an outer fluorescent lighting source, and then saved in jpeg format with a resolution of $3696 \times 2448$ pixels.

\section{2) Facial landmark detection and center point} calculation

To detect facial landmarks, we used the facial 


\begin{tabular}{|c|c|}
\hline Image acquisition & Photograph the frontal facial image of the subject \\
\hline $\begin{array}{c}\text { Facial landmarks detection } \\
\text { and center point calculation }\end{array}$ & Detect facial landmarks and calculate the center point of the landmarks \\
\hline $\begin{array}{c}\text { Normalization and feature } \\
\text { extraction }\end{array}$ & Normalize the positions of facial landmarks and calculate facial \\
morphological features
\end{tabular}

Fig. 2. Schematic diagram of facial shape feature extraction.

landmark detector included in the Dlib library ${ }^{29)}$, which implements the Kazemi and Sullivan method $^{30)}$. The detector provides 68 landmark points (see Figure 3, The original image is from the CMU Multi-PIE Face Database ${ }^{31)}$ ). The center point of a face is determined as the average of the position values of points 18 to 68 (eyebrows, eyes, nose, and mouth) on the $x$-axis and $y$-axis. The center point of a face $\overline{\mathrm{p}}=(\bar{x}, \bar{y})$ of $\mathrm{p}_{18}, \cdots, \mathrm{p}_{68}$ located at $\left(x_{18}, y_{18}\right), \cdots,\left(x_{68}, y_{68}\right)$ is calculated as follows:

$$
\bar{x}=\frac{1}{(68-17)} \sum_{i=18}^{68} x_{i} \quad \bar{y}=\frac{1}{(68-17)} \sum_{i=18}^{68} y_{i}
$$

The points 1 to 17 are the lower edge of the face and were excluded in the center point calculation because points 1 to 17 of a person with a higher BMI are located lower than those of a person with a lower BMI. This is because the higher the BMI, the higher the facial adiposity, and the fatter and bulkier the cheeks and chin become. Therefore, points 1 to 17 were excluded to set the face center to the same position regardless of BMI. (Figure 3)

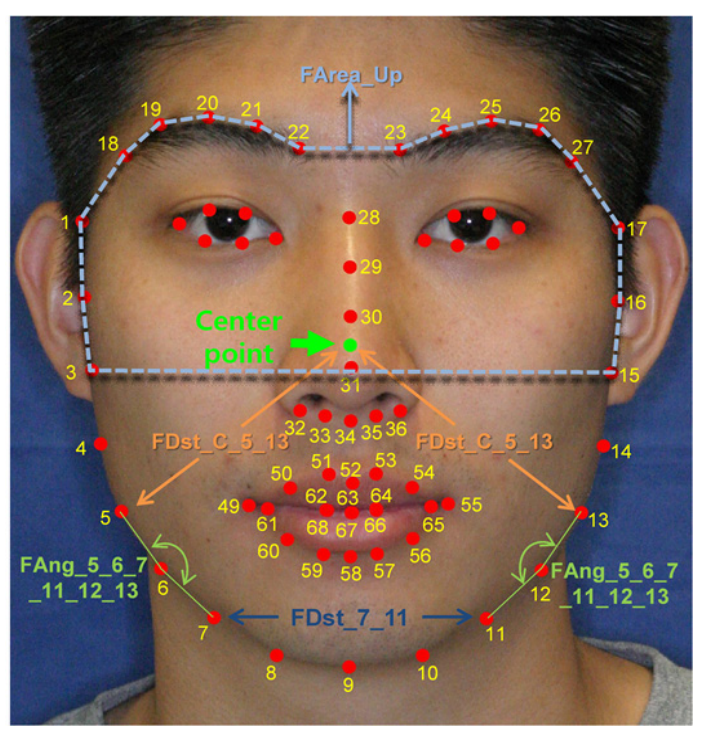

Fig. 3. An example of the fiducial points on a frontal face image. The center point is marked with green point.

The red dots denote the facial landmark points, and the numbers next to the red dots indicate the order of the points. FDst_7_11 (normalized distance between point 7 and point 11), FDst_C_5_13 (the average value of FDst_C_5 and FDst_C_13 where FDst_C 5 and FDst_C_13 are normalized distances between the center point and point 5 and the center point and point 13). FAng_5_6_7_11 12_13 (the average value of FAng_5_6_7 and FAng_11_12_13 where FAng_5_6_7 and FAng_11_12_13 are the included angles between the points 5, 6 and 7 and the points 11,12 and 13, respectively) are indicated by arrows and FArea_Up is indicated by a dotted line respectively. This original image is from the CMU Multi-PIE Face Database 45 , which is a publicly released image database. 
3) Normalization and facial morphological features calculation

Because individuals have different face sizes, the positions of facial landmarks need to be transformed so that they are not affected by the face size of each subject. Transformation is performed by translation and scaling. Translation is calculated by subtracting the position of the center point $\bar{p}$ from the position of each facial landmark, and the translated landmarks are scaled by the mean distance $M D$. That is, the $i$-th translated facial landmark $\mathrm{p}_{i}^{\prime}=\left(x_{i}^{\prime}, y_{i}^{\prime}\right)$ is determined as follows:

$$
\begin{aligned}
& x_{i}^{\prime}=\frac{x_{i}-\bar{x}}{M D} \quad y_{i}^{\prime}=\frac{y_{i}-\bar{y}}{M D} \quad \text { where } \\
& M D=\frac{1}{(68-17)} \sum_{i=18}^{68} \sqrt{\left(x_{i}-\bar{x}\right)^{2}+\left(y_{i}-\bar{y}\right)^{2}}
\end{aligned}
$$

Facial morphological features were calculated using the normalized facial landmarks and consisted of four parts: (1) the distances between two points of facial landmarks: the distance between the $i$-th and the $j$-th points was expressed as FDst_ijj. For example, FDst_7_11 was the normalized distance between point 7 and point 11; (2) the distances between the center point of a face and the facial landmark points: the distance between the center point of a face and the $i$-th and the $j$-th points was expressed as FDst_C_i_j. For example, FDst_C_5_13 was the average of the normalized distances between the center point of a face and point 5 and the center point and point 17; (3) the angles between the neighboring three landmarks on the face outline: the angle between the $i$-th, the $j$-th, the $k$-th, the $l$-th, the $m$-th, and the $n$-th points was expressed as FAng_i_j_k_1_m_n. For instance, FAng_5 6_7_11_12_13 was the average of the two angles, the included angle between the points 5 , 6 and 7 and the other included angle between the points 11, 12 and 13; (4) the areas of each part of a face: For example, FArea_Up denoted the area of the upper part of a face. The features are described in detail in Table 1 and Figure 3.

\section{Cold pattern}

The cold pattern questionnaire consisted of eight items to assess symptoms: aversion to cold temperature, preference for warm temperature, cold abdomen, cold limbs, feeling cold in the body, pale face, preference for warm water, and clear urine. All items on the self-administered questionnaire were presented with a 5-point scale from "strongly disagree" (score 1) to "strongly agree" (score 5). The sum of the eight scores is the cold pattern score (CPS), which may range from 8 to 40. The higher the CPS value, the more severe the cold pattern symptoms. Agreement between two experts for this questionnaire was $87.1 \%$ (Kappa value 0.741$)^{32)}$.

\section{Statistical analysis}

All statistical analyses were performed using SPSS 22.0 (IBM Corp., Armonk, NY, USA). All continuous variable data were reported as means \pm standard deviation and categorical data were reported as percentages. Pearson's correlation coefficients and partial correlation coefficients controlling for sex, age, and BMI were calculated to assess the degree of the association between facial features and cold pattern symptoms. The differences of sample characteristics between sexes 
were compared using a two-sample $t$-test. A $p$-value less than 0.05 was considered statistically significant. (Table 1)

\section{Results}

\section{Characteristics of participants}

This study included 185 male and 267 female participants with a mean age of $39.5 \pm 3.4$ years (range: 29.9-49.0 years). The mean CPS and mean BMI were $24.6 \pm 5.2$ (range: 9-39) and $23.6 \pm$ 3.4 (range: 15.7- 34.1), respectively as shown in
Table 2. The CPS distribution of male and female participants is shown in Figure 4.

\section{Association between facial morphological features and CPS}

The results presented in Table 3 show the statistical analysis of the facial morphological features with CPS. The results are organized as follows: (1) The distances between two points of facial landmarks; the results of a partial correlation coefficients showed that FDst_7_11 $(r=-0.102$, $p=0.030)$ and FDst $\_810(r=-0.114, p=0.015)$

Table 1. Facial morphological features and descriptions of the study

\begin{tabular}{|c|c|c|}
\hline Feature group & & Feature names and descriptions \\
\hline \multirow[t]{2}{*}{$\begin{array}{l}\text { 1) Distances between two } \\
\text { points of facial landmarks }\end{array}$} & Features & $\begin{array}{l}\text { FDst_1_17, FDst_2_16, FDst_3_15, FDst_4_14, FDst_5_13, FDst_6_12, } \\
\text { FDst_7_11, FDst_8_10 }\end{array}$ \\
\hline & Descriptions & $\begin{array}{l}\text { FDst_1_17: Normalized distance between point } 1 \text { and point } 17 \text {. The others are } \\
\text { described in the same way. }\end{array}$ \\
\hline \multirow[t]{2}{*}{$\begin{array}{l}\text { 2) Distances between the center } \\
\text { point of a face and the facial } \\
\text { landmark points }\end{array}$} & Features & $\begin{array}{l}\text { FDst_C_1_17, FDst_C_2 } 16 \text {, FDst_C_3 } 315 \text {, FDst_C_4 } 14 \text {, FDst_C_5 } 13 \text {, } \\
\text { FDst_C_6_12, FDst_C_7 } 11 \text {, FDst_C_8_10, FDst_C_37_46, FDst_C_38_45, } \\
\text { FDst_C_39_44, FDst_C_40_43, FDst_C_41_48, FDst_C_42_47, FDst_C_49_55, } \\
\text { FDst_C_50_54, FDst_C_51_53, FDst_C_60_56, FDst_C_59_57, FDst_C_61_65, } \\
\text { FDst_C_62_64, FDst_C_68_66 }\end{array}$ \\
\hline & Descriptions & $\begin{array}{l}\text { FDst_C_1_17 }=(\text { FDst_C_1 }+ \text { FDst_C_17) } / 2 \text {, where FDst_C_1 and FDst_C_17 are } \\
\text { the normalized distances between the center point } \bar{p} \text { and point } 1\end{array}$ \\
\hline \multirow{2}{*}{$\begin{array}{l}\text { 3) Angles between the } \\
\text { neighboring three landmarks } \\
\text { on the face outline and the } \\
\text { center point } \overline{\mathrm{p}} \text { and point } 17 \text {, } \\
\text { respectively. The others are } \\
\text { described in the same way. }\end{array}$} & Features & 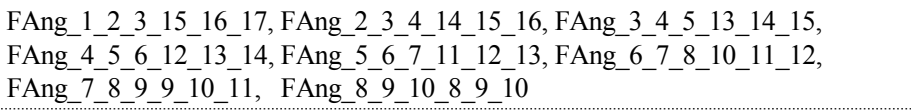 \\
\hline & Descriptions & $\begin{array}{l}\text { FAng } 11233 \_15 \_16 \_17=\left(\text { FAng } 1 \_2 \_3+\text { FAng } 15 \_16 \_17\right) / 2 \text {, where FAng_1_2_3 } \\
\text { is the included angle between the line connecting point } 2 \text { and point } 1 \text { and the line } \\
\text { connecting point } 2 \text { and point } 3 \text {. The others are described in the same way. }\end{array}$ \\
\hline \multirow[t]{8}{*}{ 4) Areas of each part of a face } & Features & FArea_Up \\
\hline & Descriptions & $\begin{array}{l}\text { The area of the upper part of the face. The area of the region enclosed by points } 1,18 \text {, } \\
19,20,21,22,23,24,25,26,27,17,16,15,3 \text {, and } 2 \text {. }\end{array}$ \\
\hline & Features & FArea_Down \\
\hline & Descriptions & $\begin{array}{l}\text { The area of the lower part of the face. The area of the region enclosed by the points } 3 \text {, } \\
15,14,13,12,11,10,9,8,7,6,5 \text {, and } 4 \text {. }\end{array}$ \\
\hline & Features & FArea_In \\
\hline & Descriptions & $\begin{array}{l}\text { The area of the sensory organs of the face. The area of the region enclosed by the } \\
\text { points from } 18 \text { to } 68 \text {. }\end{array}$ \\
\hline & Features & FArea_Out \\
\hline & Descriptions & $\begin{array}{l}\text { The area of the face excluding the sensory organs. Full face area (the area of the region } \\
\text { enclosed by points } 1 \text { to } 68 \text { ) excluding FArea_In. }\end{array}$ \\
\hline
\end{tabular}




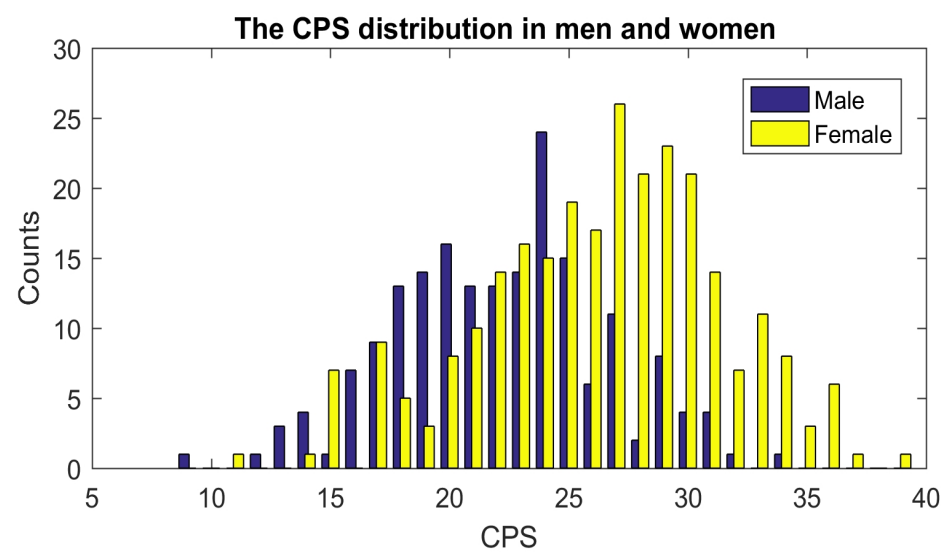

Fig. 4. The CPS distribution of male and female participants.

were negatively associated with CPS. (2) The distances between the center point of a face and the facial landmark points; while the distance features of FDst_C_5_13 $(r=-0.106, p=0.025)$, FDst_C_6_12 $(r=-0.112, p=0.017)$, FDst_C_7_11 $(r=-0.103, p=0.029)$, FDst_C_49_55 $(r=-0.097$, $p=0.040)$, and FDst_C_61_65 $(r=-0.113, p=$ $0.017)$ were negatively associated with CPS, the distance features of FDst_C_40_43 $(r=0.110, p$ $=0.020)$, FDst_C_41_48 $(r=0.118, p=0.012)$, and FDst_C_42_47 $(r=0.104, p=0.028)$ were positively associated with CPS. (3) The angles between the neighboring three landmarks on the face outline; FAng 1_23_15_16_17 $(r=0.105, p=$ 0.026) and FAng_7_8_9_9_10_11 $(r=0.098, p=$
0.038) were positively associated with CPS, whereas, FAng_5_6_7_11_12_13 $(r=-0.094, p$ $=0.047)$ were negatively associated with CPS. (4) The areas of each part of a face; the partial correlation coefficients results showed that FArea_Up $(r=-0.117, p=0.013)$ and FArea_Out $(r$ $=-0.095, p=0.044)$ were negatively associated with CPS. The number of face morphological features significant with CPS was 37 (the statistically corrected results were 15). (Table 3)

The significant difference between facial morphological features and CPS may be due to gender differences since, in general, women tend to have higher CPS than men. Therefore, statistical significance was analyzed according to sex.

Table 2. Characteristics of participants

\begin{tabular}{lcccc}
\hline \multirow{2}{*}{ Number of participants } & \multirow{2}{*}{452} & Male & Female & \multirow{2}{*}{$p$-value } \\
\cline { 3 - 4 } & & $185(40.9 \%)$ & $267(59.1 \%)$ & 0.003 \\
Age (year) & $39.5 \pm 3.4$ & $39.0 \pm 3.3$ & $39.9 \pm 3.4$ & $<0.001$ \\
CPS & $24.6 \pm 5.2$ & $22.2 \pm 4.4$ & $26.3 \pm 5.1$ & $<0.001$ \\
BMI & $23.6 \pm 3.4$ & $25.3 \pm 3.1$ & $22.4 \pm 3.1$ & \\
\hline
\end{tabular}

Statistically significant differences between group means as determined by two-sample t-test are in bold; CPS, cold pattern scores; BMl, body mass index 
Table 4 and Table 5 show the statistical analysis results between facial morphological features and CPS for male and female participants, respectively. The number of facial morphological features that were statistically significant with CPS of male participants was 27 (statistically corrected results were 0 ). In the case of female participants, the number of features statistically significant with CPS was 33 (statistically corrected results were 15). Female participants had 6 more statistically significant features than male participants, and after adjusting for age and BMI, there were 15 more. The differences between female and male participants were as follows: the distance features of FDst_C_38_45 $(r=0.164, p=0.007)$, FDst C_39_44 $(r=0.195, p=0.001)$, FDst_C_40_43 $(r=$ $0.214, p=0.000)$, FDst_C_41_48 $(r=0.202, p=$ $0.001)$, and FDst_C_42_47 $(r=0.155, p=$ 0.011 ) were positively associated with CPS in female participants, however, that of the male participants was not significant. In other words, in female participants, the higher the CPS, the farther the distance between the central point of the face and the eyes. Overall, it was found that the proposed facial morphological features were more suitable for predicting female participants' CPS. (Table 4, 5)

Table 3. Correlation coefficients between facial morphological features and CPS for all participants

\begin{tabular}{|c|c|c|c|c|c|c|c|}
\hline & Features & Unadjusted & Adjusted & & Features & Unadjusted & Adjusted \\
\hline \multirow[t]{8}{*}{1} & FDst_1_17 & $-0.249^{* *}$ & -0.026 & \multirow[t]{9}{*}{2} & FDst_C_42_47 & $0.125^{* *}$ & $0.104^{*}$ \\
\hline & FDst_2_16 & $-0.280^{* *}$ & -0.019 & & FDst_C_49_55 & $-0.291^{* *}$ & $-0.097^{*}$ \\
\hline & FDst_3_15 & $-0.334^{* * *}$ & -0.024 & & FDst_C_50_54 & $-0.095^{*}$ & -0.033 \\
\hline & FDst_4_14 & $-0.363^{* *}$ & -0.047 & & FDst_C_51_53 & 0.044 & 0.025 \\
\hline & FDst_5_13 & $-0.370^{* * *}$ & -0.066 & & FDst_C_60_56 & -0.044 & -0.060 \\
\hline & FDst_6_12 & $-0.367^{* *}$ & -0.091 & & FDst_C_59_57 & $0.104^{*}$ & -0.022 \\
\hline & FDst_7_11 & $-0.367^{* *}$ & $-0.102^{*}$ & & FDst_C_61_65 & $-0.288^{* *}$ & $-0.113^{*}$ \\
\hline & FDst_8_10 & $-0.368^{* * *}$ & $-0.114^{*}$ & & FDst_C_62_64 & 0.070 & -0.012 \\
\hline \multirow[t]{13}{*}{2} & FDst_C_1_17 & $-0.161^{* *}$ & 0.042 & & FDst_C_68_66 & $0.147^{* *}$ & 0.025 \\
\hline & FDst_C_2_16 & $-0.263^{* * *}$ & 0.006 & \multirow[t]{8}{*}{3} & FAng_1_2_3_15_16_17 & $0.352^{* * *}$ & $0.105^{*}$ \\
\hline & FDst_C_3_15 & $-0.341^{* *}$ & -0.045 & & FAng_2_3_4_14_15_16 & $-0.149^{* *}$ & 0.064 \\
\hline & FDst_C_4_14 & $-0.359^{* * *}$ & -0.085 & & FAng_3_4_5_13_14_15 & $-0.236^{* * *}$ & -0.031 \\
\hline & FDst_C_5_13 & $-0.362^{* *}$ & $-0.106^{*}$ & & FAng_4_5_6_12_13_14 & $-0.257^{* *}$ & -0.068 \\
\hline & FDst_C_6_12 & $-0.353^{* *}$ & $-0.112^{*}$ & & FAng $5 \_6 \_711 \_12 \_13$ & $-0.147^{* *}$ & $-0.094^{*}$ \\
\hline & FDst_C_7_11 & $-0.335^{* *}$ & $-0.103^{*}$ & & FAng_6_788_10_11_12 & 0.087 & 0.005 \\
\hline & FDst_C_8_10 & $-0.300^{* *}$ & -0.079 & & FAng 7_8_9_9_10_11 & $0.171^{* *}$ & $0.098^{*}$ \\
\hline & FDst_C_37_46 & $0.101^{*}$ & 0.081 & & FAng_8_9_10_8_9_10 & $0.250^{* *}$ & 0.067 \\
\hline & FDst_C_38_45 & $0.230^{* *}$ & 0.083 & \multirow[t]{4}{*}{4} & FArea_Up & $-0.296^{* *}$ & $-0.117^{*}$ \\
\hline & FDst_C_39_44 & $0.245^{* *}$ & 0.087 & & FArea_Down & $-0.293^{* *}$ & 0.006 \\
\hline & FDst_C_40_43 & 0.059 & $0.110^{*}$ & & FArea_In & $-0.238^{* * *}$ & -0.056 \\
\hline & FDst_C_41_48 & $0.105^{*}$ & $0.118^{*}$ & & FArea_Out & $-0.386^{* *}$ & $-0.095^{*}$ \\
\hline
\end{tabular}

Unadjusted, Pearson's correlation coefficients; adjusted, partial correlation coefficients adjusted for sex, age, and BMI.

${ }^{*} \not\left\langle 0.05 ;{ }^{* *} \not\langle 0.01\right.$. 1, the features of distance between two points of facial landmarks; 2 , the features of distance between the center point of a face and the facial landmark points; 3 , the angle features; 4 , the area features. 


\section{Association between facial morphological features and specific cold pattern symptoms}

Table 6 shows the statistical analysis results of the facial morphological features compared with specific cold pattern symptoms. FDst_C_1_17 value (the average of the normalized distances between the center point $\overline{\mathrm{p}}$ and point 1 and the center point $\overline{\mathrm{p}}$ and point 17) was significantly correlated with cold abdomen $(r=0.109, p=$ 0.021). FDst_C_5_13 value was significantly correlated with aversion to cold $(r=-0.095, p=$ $0.045)$ and cold limbs $(r=-0.107, p=0.023)$. FDst_C_6_12 and FDst_C_7_11 values were significantly correlated with cold limbs (FDst_C_6_12: $r=-0.113, p=0.017$; FDst_C_7_11: $r=-0.107, p=0.023)$. FDst_C_39_44 value was significantly correlated with aversion to cold temperature $(r=0.110, p=0.020)$. FDst_C_40_43 value was significantly correlated with aversion to cold temperature $(r=0.148, p=0.002)$, cold limbs $(r=0.126, p=0.007)$, and feeling cold in the body $(r=0.096, p=0.041)$. FDst_C_41_48 value was significantly correlated with aversion to cold ( $r=0.124, p=0.008)$, cold limbs $(r=$ 0.097, $p=0.040$ ), and feeling cold in the body $(r=0.103, p=0.029)$. FDst_C_42_47 value was significantly correlated with aversion to cold $(r=$

Table 4. Correlation coefficients between facial morphological features and CPS for male participants

\begin{tabular}{|c|c|c|c|c|c|c|c|}
\hline & Features & Unadjusted & Adjusted & & Features & Unadjusted & Adjusted \\
\hline \multirow[t]{8}{*}{1} & FDst_1_17 & $-0.227^{* *}$ & 0.012 & \multirow[t]{9}{*}{2} & FDst_C_42_47 & 0.038 & 0.056 \\
\hline & FDst_2_16 & $-0.256^{* *}$ & -0.002 & & FDst_C_49_55 & $-0.225^{* *}$ & -0.063 \\
\hline & FDst_3_15 & $-0.288^{* * *}$ & -0.011 & & FDst_C_50_54 & -0.006 & -0.018 \\
\hline & FDst_4_14 & $-0.296^{* *}$ & -0.019 & & FDst_C_51_53 & 0.118 & 0.027 \\
\hline & FDst_5_13 & $-0.276^{* *}$ & -0.013 & & FDst_C_60_56 & -0.075 & -0.091 \\
\hline & FDst_6_12 & $-0.250^{* * *}$ & -0.008 & & FDst_C_59_57 & -0.009 & -0.105 \\
\hline & FDst_7_11 & $-0.205^{* *}$ & -0.010 & & FDst_C_61_65 & $-0.209^{* * *}$ & -0.087 \\
\hline & FDst_8_10 & $-0.182^{*}$ & -0.030 & & FDst_C_62_64 & 0.024 & -0.085 \\
\hline \multirow[t]{13}{*}{2} & FDst_C_1_17 & -0.134 & 0.061 & & FDst_C_68_66 & 0.037 & -0.054 \\
\hline & FDst_C_2_16 & $-0.236^{* *}$ & 0.018 & \multirow[t]{8}{*}{3} & FAng_1_2_3_15_16_17 & $0.202^{* *}$ & 0.022 \\
\hline & FDst_C_3_15 & $-0.292^{* *}$ & -0.024 & & FAng_2_3_4_14_15_16 & -0.123 & 0.016 \\
\hline & FDst_C_4_14 & $-0.300^{* *}$ & -0.051 & & FAng 3_455_13_14_15 & $-0.239^{* *}$ & -0.058 \\
\hline & FDst_C_5_13 & $-0.294^{* * *}$ & -0.064 & & FAng_4_5_6_12_13_14 & $-0.186^{*}$ & -0.029 \\
\hline & FDst_C_6_12 & $-0.284^{* *}$ & -0.072 & & FAng_5_6_7_11_12_13 & $-0.156^{*}$ & 0.031 \\
\hline & FDst_C_7_11 & $-0.260^{* *}$ & -0.092 & & FAng_6_7_8_10_11_12 & $0.229^{* *}$ & 0.017 \\
\hline & FDst_C_8_10 & $-0.240^{* *}$ & -0.109 & & FAng_7_8_9_9_10_11 & $0.213^{* *}$ & 0.016 \\
\hline & FDst_C_37_46 & 0.028 & 0.074 & & FAng_8_9_10_8_9_10 & 0.067 & -0.019 \\
\hline & FDst_C_38_45 & 0.073 & 0.041 & \multirow[t]{4}{*}{4} & FArea_Up & $-0.260^{* *}$ & -0.056 \\
\hline & FDst_C_39_44 & 0.071 & 0.006 & & FArea_Down & $-0.192^{* *}$ & 0.001 \\
\hline & FDst_C_40_43 & 0.074 & -0.022 & & FArea_In & $-0.178^{*}$ & 0.020 \\
\hline & FDst C 4148 & 0.045 & 0.015 & & FArea Out & $-0.321^{* *}$ & -0.071 \\
\hline
\end{tabular}

Unadjusted, Pearson's correlation coefficients; adjusted, partial correlation coefficients adjusted for sex, age, and BMI. " $\not\left\langle 0.05 ;{ }^{* *} \not\langle 0.01\right.$. 1, the features of distance between two points of facial landmarks; 2 , the features of distance between the center point of a face and the facial landmark points; 3 , the angle features; 4 , the area features. 
0.095, $p=0.043)$, feeling cold in the body $(r=$ $0.099, p=0.037)$, and preference for warm water $(r=0.095, p=0.044)$. FDst_C_49_55 value was significantly correlated with aversion to cold $(r=$ -0.096, $p=0.042)$, cold limbs $(r=-0.105, p=$ $0.026)$, and preference for warm water $(r=$ $-0.103, p=0.029)$. FDst_C_61_65 value was significantly correlated with aversion to cold $(r=$ -0.100, $p=0.035)$, cold limbs $(r=-0.108, p=$ $0.022)$, and preference for warm water $(r=$ -0.111, $p=0.019$ ).

FAng_1_2_3_15_16_17 value was significantly correlated with aversion to cold $(r=0.108, p=$ $0.023)$ and cold limbs $(r=0.100, p=0.035)$.
FAng_2_3_4_14_15_16 value was significantly correlated with cold abdomen $(r=0.108, p=$ $0.023)$ and pale face $(r=0.093, p=0.049)$. FAng_4_5_6_12_13_14 value was significantly correlated with aversion to cold $(r=-0.106, p=$ 0.024). FAng_7_8_9_9_10_11 value was significantly correlated with aversion to cold $(r=0.123, p=$ $0.009)$ and cold limbs ( $r=0.111, p=0.019)$.

FArea_Up value was significantly correlated with aversion to cold ( $r=-0.093, p=0.049$ ) and cold limbs $(r=-0.116, p=0.014)$. FArea _Down value was significantly correlated with preference for warm temperature $(r=-0.093, p=$ 0.048). FArea_Out value was significantly

Table 5. Correlation coefficients between facial morphological features and CPS for female participants

\begin{tabular}{|c|c|c|c|c|c|c|c|}
\hline & Features & Unadjusted & Adjusted & & Features & Unadjusted & Adjusted \\
\hline \multirow[t]{8}{*}{1} & FDst_1_17 & $-0.201^{* *}$ & -0.047 & \multirow[t]{9}{*}{2} & FDst_C_42_47 & $0.155^{*}$ & $0.134^{*}$ \\
\hline & FDst_2_16 & $-0.197^{* *}$ & -0.026 & & FDst_C_49_55 & $-0.196^{* *}$ & -0.115 \\
\hline & FDst_3_15 & $-0.215^{* *}$ & -0.027 & & FDst_C_50_54 & -0.055 & -0.043 \\
\hline & FDst_4_14 & $-0.246^{* *}$ & -0.059 & & FDst_C_51_53 & 0.042 & 0.022 \\
\hline & FDst_5_13 & $-0.263^{* *}$ & -0.096 & & FDst_C_60_56 & -0.033 & -0.047 \\
\hline & FDst_6_12 & $-0.283^{* *}$ & $-0.141^{*}$ & & FDst_C_59_57 & 0.072 & 0.023 \\
\hline & FDst_7_11 & $-0.279^{* *}$ & $-0.159^{* *}$ & & FDst_C_61_65 & $-0.194^{* *}$ & $-0.128^{*}$ \\
\hline & FDst_8_10 & $-0.272^{* *}$ & $-0.168^{* *}$ & & FDst_C_62_64 & 0.039 & 0.026 \\
\hline \multirow[t]{13}{*}{2} & FDst_C_1_17 & -0.103 & 0.031 & & FDst_C_68_66 & $0.126^{*}$ & 0.070 \\
\hline & FDst_C_2_16 & $-0.171^{* *}$ & 0.002 & \multirow[t]{8}{*}{3} & FAng $1223315 \_16 \_17$ & $0.245^{* *}$ & $0.141^{*}$ \\
\hline & FDst_C_3_15 & $-0.236^{* *}$ & -0.054 & & FAng_2_3_4_14_15_16 & 0.015 & 0.099 \\
\hline & FDst_C_4_14 & $-0.271^{* *}$ & -0.104 & & FAng_3_4_5_13_14_15 & $-0.141^{*}$ & 0.000 \\
\hline & FDst_C_5_13 & $-0.281^{* *}$ & -0.131 & & FAng_4_5_6_12_13_14 & $-0.171^{* *}$ & -0.087 \\
\hline & FDst_C_6_12 & $-0.271^{* *}$ & -0.135 & & FAng 5_6_7_11_12_13 & $-0.204^{* *}$ & $-0.150^{* *}$ \\
\hline & FDst_C_7_11 & $-0.23^{* *}$ & -0.108 & & FAng $6 \_7 \_8 \_10 \_11 \_12$ & 0.087 & -0.012 \\
\hline & FDst_C_ $8 \_10$ & $-0.177^{* *}$ & -0.061 & & FAng_7_8_9_9_10_11 & $0.219^{* *}$ & $0.141^{*}$ \\
\hline & FDst_C_37_46 & 0.095 & 0.088 & & FAng 8 8 $9 \_10 \_8 \_9 \_10$ & $0.163^{* *}$ & $0.124^{*}$ \\
\hline & FDst_C_38_45 & $0.164^{* *}$ & 0.109 & \multirow[t]{4}{*}{4} & FArea_Up & $-0.276^{* *}$ & $-0.153^{*}$ \\
\hline & FDst_C_ $39 \_44$ & $0.195^{* *}$ & $0.131^{*}$ & & FArea_Down & $-0.141^{*}$ & 0.009 \\
\hline & FDst_C_ $40 \_43$ & $0.214^{* *}$ & $0.173^{* *}$ & & FArea_In & $-0.192^{* *}$ & -0.101 \\
\hline & FDst_C_41_48 & $0.202^{* *}$ & $0.173^{* *}$ & & FArea_Out & $-0.281^{* *}$ & -0.105 \\
\hline
\end{tabular}

Unadjusted, Pearson's correlation coefficients; adjusted, partial correlation coefficients adjusted for age and BML. ${ }^{*} \not<0.05 ;{ }^{* *} \not<0.01$. 1, the features of distance between two points of facial landmarks; 2 , the features of distance between the center point of a face and the facial landmark points; 3 , the angle features; 4 , the area features. 
correlated with aversion to cold $(r=-0.095, p=$ $0.045)$ and cold limbs $(r=-0.100, p=0.033)$. (Table 6)

\section{Discussion}

In our study, we found that facial morphological features were associated with CPS even after adjustment for sex, age, and BMI. Several studies have been conducted to develop an objective and reliable pattern identification system, since the disadvantage of diagnosis based on a practitioner's subjective experience and personal knowledge leads to inconsistent diagnostic results. The features used for objective pattern identification are facial color $^{33,34)}$, increased leptin levels ${ }^{35)}$, anthropometric measures $^{36)}$, resting metabolic rate ${ }^{5)}$, heart rate variability parameters ${ }^{7}$, levels of circulating $\operatorname{adiponectin}^{37)}$, the sympathetic nervous system and glsucose metabolism ${ }^{6}$, and tongue ${ }^{38)}$. The studies on the relationship between cold pattern and facial complexion (color) ${ }^{33,34)}$ used the method that divided a face into parts and extracted the $L^{*} a^{*} b^{*}$ color values of each part and investigated the correlation with the cold pattern. Although traditional pattern identification using facial complexion is conducted with facial color ${ }^{4}$, these color-based approaches share the problem that color is typically vulnerable to environmental illumination changes ${ }^{39)}$. The proposed facial-morphological feature based pattern identification system is free from illumination conditions. Moreover, there have been no studies on the direct correlation between cold pattern and facial shape. This study was the first to objectively investigate the correlation between the facial morphological features and cold pattern.

The facial parts showing significant correlation according to cold pattern severity are the eyes, mouth, jaw, chin, and all sensory organs of the face. For the eyes, FDst_C_40_43, FDst_C_41_48, and FDst_C_42_47, which are the distances from the center point of the face to the landmarks on the eyes, were positively correlated with CPS $(P<0.05)$, and the other three distances (FDst_C 37_46, FDst_C_38_45, and FDst_C_39_44) were positively correlated with CPS and statistically quasi-significant $(P<0.09)$. This means that people with a high CPS have higher eyes.

In the mouth area, FDst_C_49_55 and FDst_C_61_65 have a negative correlation with CPS, which means that those with a high CPS have more upturned corners of the mouth.

FDst_C_5_13, FDst_C_6_12, and FDst_C_7_11 values were negatively associated with CPS, which means that the higher the CPS, the closer the jaw is to the center of the face and that the facial shape is relatively long. FAng 5_6_7_11_12_13 value was negatively correlated with $\mathrm{CPS}$, which means that the higher the CPS, the narrower the angle of the jaw; the jaws of subjects with high CPS were angled and the jaws of subjects with low CPS were rounded.

The normalized distances between points 7 and 11 and between points 8 and 10 were negatively associated with CPS. This means the subjects with high CPS have a sharp chin. FAng_7_8_ 9_9_10_11 was positively correlated with CPS, which means that the higher the CPS, the closer the lines of points 7-8-9 and 9-10-11 are to being relatively straight. This means that a person with higher CPS has a pointed chin and a 
Table 6. Partial correlation coefficients between facial morphological features and specific cold pattern symptoms for all participants

\begin{tabular}{|c|c|c|c|c|c|c|c|c|c|}
\hline & Features & $\begin{array}{l}\text { aversion to } \\
\text { cold } \\
\text { temperature }\end{array}$ & $\begin{array}{l}\text { preference for } \\
\text { warm } \\
\text { temperature }\end{array}$ & $\begin{array}{c}\text { cold } \\
\text { abdomen }\end{array}$ & $\begin{array}{l}\text { cold } \\
\text { limbs }\end{array}$ & $\begin{array}{l}\text { feeling } \\
\text { cold in the } \\
\text { body }\end{array}$ & pale face & $\begin{array}{l}\text { preference for } \\
\text { warm water }\end{array}$ & $\begin{array}{l}\text { clear } \\
\text { urine }\end{array}$ \\
\hline \multirow[t]{8}{*}{1} & FDst_1_17 & -0.044 & -0.018 & 0.043 & -0.042 & -0.010 & -0.012 & 0.006 & -0.026 \\
\hline & FDst_2_16 & -0.046 & -0.017 & 0.054 & -0.035 & 0.004 & -0.006 & 0.004 & -0.034 \\
\hline & FDst_3_15 & -0.062 & -0.020 & 0.061 & -0.038 & 0.008 & -0.002 & -0.007 & -0.036 \\
\hline & FDst_4_14 & -0.076 & -0.029 & 0.043 & -0.056 & -0.004 & -0.014 & -0.018 & -0.039 \\
\hline & FDst_5_13 & -0.087 & -0.034 & 0.022 & -0.070 & -0.018 & -0.015 & -0.024 & -0.047 \\
\hline & FDst_6_12 & -0.091 & -0.047 & -0.002 & -0.081 & -0.036 & -0.011 & -0.048 & -0.058 \\
\hline & FDst_7_11 & -0.081 & -0.069 & -0.011 & -0.084 & -0.043 & 0.006 & -0.061 & -0.076 \\
\hline & FDst_8_10 & -0.069 & -0.083 & -0.023 & -0.086 & -0.049 & -0.005 & -0.079 & -0.078 \\
\hline \multirow[t]{22}{*}{2} & FDst_C_1_17 & 0.011 & -0.044 & $0.109^{*}$ & 0.012 & 0.028 & 0.008 & 0.063 & -0.019 \\
\hline & FDst_C_2_16 & -0.024 & -0.023 & 0.081 & -0.019 & 0.017 & 0.002 & 0.024 & -0.033 \\
\hline & FDst_C 315 & -0.067 & -0.011 & 0.033 & -0.058 & -0.009 & -0.010 & -0.026 & -0.033 \\
\hline & FDst_C_4_14 & -0.087 & -0.013 & -0.012 & -0.089 & -0.035 & -0.025 & -0.051 & -0.031 \\
\hline & FDst_C_5_13 & $-0.095^{*}$ & -0.019 & -0.038 & $-0.107^{*}$ & -0.048 & -0.022 & -0.061 & -0.039 \\
\hline & FDst_C_6_12 & -0.083 & -0.031 & -0.049 & $-0.113^{*}$ & -0.050 & -0.001 & -0.077 & -0.050 \\
\hline & FDst_C_7_11 & -0.053 & -0.052 & -0.050 & $-0.107^{*}$ & -0.044 & 0.027 & -0.076 & -0.061 \\
\hline & FDst_C_8_10 & -0.018 & -0.067 & -0.044 & -0.084 & -0.024 & 0.043 & -0.066 & -0.060 \\
\hline & FDst_C_37_46 & 0.070 & 0.002 & 0.042 & 0.036 & 0.078 & 0.016 & 0.085 & -0.006 \\
\hline & FDst_C_38_45 & 0.084 & 0.006 & 0.012 & 0.065 & 0.085 & -0.030 & 0.090 & 0.014 \\
\hline & FDst_C_39_44 & $0.110^{*}$ & 0.025 & -0.008 & 0.090 & 0.084 & -0.033 & 0.070 & 0.004 \\
\hline & FDst_C_40_43 & $0.148^{* *}$ & 0.017 & 0.006 & $0.126^{* *}$ & $0.096^{*}$ & 0.011 & 0.049 & -0.022 \\
\hline & FDst_C_41_48 & $0.124^{* *}$ & 0.022 & 0.018 & $0.097^{*}$ & $0.103^{*}$ & 0.048 & 0.086 & -0.030 \\
\hline & FDst_C_42_47 & $0.095^{*}$ & 0.008 & 0.030 & 0.068 & $0.099^{*}$ & 0.032 & $0.095^{*}$ & -0.013 \\
\hline & FDst_C_49_55 & $-0.096^{*}$ & -0.014 & -0.014 & $-0.105^{*}$ & -0.031 & 0.028 & $-0.103^{*}$ & -0.052 \\
\hline & FDst_C $50 \_54$ & -0.018 & 0.083 & -0.067 & 0.002 & -0.059 & -0.053 & -0.064 & 0.050 \\
\hline & FDst_C_51_53 & 0.014 & 0.071 & -0.009 & 0.048 & -0.050 & -0.055 & 0.008 & 0.080 \\
\hline & FDst_C_60_56 & -0.018 & -0.081 & -0.015 & -0.021 & 0.018 & -0.011 & -0.085 & -0.040 \\
\hline & FDst_C_59_57 & 0.024 & -0.070 & -0.013 & 0.009 & 0.030 & -0.024 & -0.042 & -0.013 \\
\hline & FDst_C_61_65 & $-0.100^{*}$ & -0.014 & -0.038 & $-0.108^{*}$ & -0.044 & -0.001 & $-0.111^{*}$ & -0.037 \\
\hline & FDst_C_62_64 & -0.050 & 0.025 & -0.032 & 0.028 & -0.028 & -0.027 & -0.011 & 0.050 \\
\hline & FDst_C_68_66 & 0.058 & -0.015 & 0.015 & 0.019 & 0.040 & -0.016 & -0.008 & 0.004 \\
\hline \multirow[t]{8}{*}{3} & FAng_1_2_3_15_16_17 & $0.108^{*}$ & 0.035 & -0.012 & $0.100^{*}$ & 0.055 & 0.034 & 0.078 & 0.025 \\
\hline & FAng 2 3 4 14_15 16 & -0.039 & 0.016 & $0.108^{*}$ & 0.037 & 0.065 & $0.093^{*}$ & -0.016 & 0.012 \\
\hline & FAng 3_4_5_13_14_15 & -0.030 & -0.009 & -0.002 & -0.033 & 0.001 & -0.055 & -0.026 & 0.029 \\
\hline & FAng $4 \_56612 \_13 \_14$ & $-0.106^{*}$ & 0.030 & -0.044 & -0.080 & -0.040 & -0.078 & 0.037 & 0.004 \\
\hline & FAng_5_6_7_11_12_13 & -0.070 & 0.003 & -0.053 & -0.085 & -0.050 & -0.004 & -0.083 & -0.033 \\
\hline & FAng_6_7_8_10_11_12 & 0.042 & -0.042 & 0.022 & -0.002 & -0.018 & 0.075 & 0.006 & -0.061 \\
\hline & FAng $7 \_899910 \_11$ & $0.123^{* *}$ & -0.033 & 0.066 & $0.111^{*}$ & 0.066 & 0.018 & 0.030 & 0.010 \\
\hline & FAng $8 \_9 \_10 \_8 \_9 \_10$ & 0.021 & 0.004 & -0.083 & -0.061 & -0.002 & -0.034 & 0.041 & 0.068 \\
\hline \multirow[t]{4}{*}{4} & FArea_Up & $-0.093^{*}$ & -0.002 & -0.057 & $-0.116^{*}$ & -0.068 & -0.033 & -0.080 & -0.019 \\
\hline & FArea_Down & -0.008 & $-0.093^{*}$ & 0.092 & -0.012 & 0.032 & 0.050 & 0.022 & -0.064 \\
\hline & FArea_In & -0.021 & 0.003 & -0.068 & -0.072 & -0.028 & -0.011 & -0.022 & -0.008 \\
\hline & FArea_Out & $-0.095^{*}$ & -0.069 & 0.034 & $-0.100^{*}$ & -0.038 & 0.005 & -0.060 & -0.062 \\
\hline
\end{tabular}

Partial correlation coefficients adjusted for gender, age, and BMl. Statistically significant results are in bold. " $P\left(0.05 ;{ }^{* *} P\langle 0.01\right.$; 


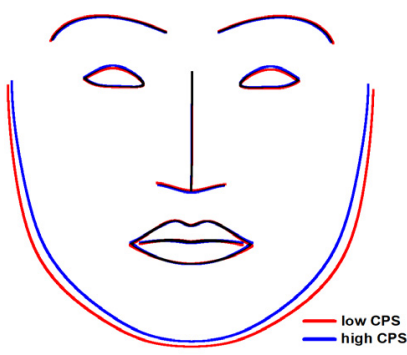

(a)

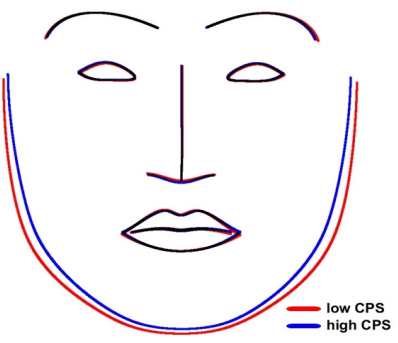

(b)

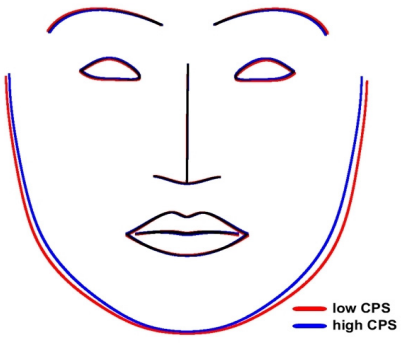

(c)

Fig. 5. Comparison of facial shapes of individuals with a high cold pattern score (CPS) (shown in blue) and individuals with a low CPS (shown in red). Individuals with a high CPS have higher eyes, a narrower angle of the jaw, more upturned corners of the mouth, and a smaller upper area of the face. (a) all participants (b) male participants (c) female participants

person with a lower score has a rounded chin.

For all sensory organs of the face, the FArea_Up value has a negative correlation with CPS, which means that the lower the CPS, the larger the relative upper area of the face. The FArea_Out value is also negatively correlated with the CPS, which means that the lower the CPS, the more small and centrally located the sensory organs of the face.

Figure 5 compares the facial contours of individuals with a high CPS (shown in blue; subjects with higher CPS scores than the third quartile; 113 in total) and those with a lower CPS (shown in red; subjects with lower CPS scores than the first quartile; 103 in total). The differences in facial morphological features mentioned above can be seen in the Figure 5.

Although the exact biological basis for cold pattern remains unclear, it is assumed that cold pattern is associated with reduced levels of thyroid $^{8)}$ or adrenal hormones ${ }^{10)}$ or decreased metabolic rate ${ }^{5)}$.

Adrenal hormones can be a major cause ${ }^{10)}$.
Adrenal fatigue or adrenal stress can cause an imbalance of hormones such as cortisol or dehydroepiandrosterone. This imbalance of adrenal hormones can lead to cold intolerance, feeling tired, sleepiness, dry skin, and weight gain. There has been no study on whether adrenal hormone directly affects changes in facial shape. However, Windhager et al. reported that cortisol correlates with changes in facial shape ${ }^{16)}$. In that study, they investigated the association between male facial shape and body mass index (BMI), salivary cortisol, and perceived health. Their results showed that men with lower salivary cortisol levels had a longer facial outline than men with higher levels. Men with low salivary cortisol levels also had higher eyebrows, a longer nose, fuller lips, upturned corners of the mouth, and a more pointed chin. In addition, the face sensory organs of men with low salivary cortisol were relatively widespread. The facial morphological features of men with low levels of salivary cortisol are very similar to those of high CPS subjects. The fact that subjects with 
low salivary cortisol levels and those with high CPS have a similar facial shape supports the hypothesis that decreased hormones or diminished functioning of the whole body increases cold sensitivity.

Since cold pattern comprises a set of multiple symptoms or signs, it needs to be diagnosed comprehensively. Therefore, the association between facial morphological features and specific symptoms was investigated. The facial morphological features that were significantly correlated with each symptom were different from the facial morphological features that were significantly correlated with CPS. The numbers of significant facial morphological features were different for each symptom; aversion to cold temperatures had the most number of significant features, and clear urine had the least number of significant features.

This study has certain limitations that should be considered. First, this study was performed on only 452 subjects, which were all in their thirties or forties. Second, this study only examined Korean adult subjects, and the results may slightly differ from other ethnic groups. Third, we used a self-report cold pattern questionnaire based on usual symptoms ${ }^{32,40)}$, and did not perform other cold pattern questionnaire or a comprehensive inspection by a practitioner. Therefore, even though the questionnaire used in this study was developed based on the previously developed cold pattern questionnaires and expert's evaluation, it cannot be confirmed in this study whether it is applicable to the disease-based pattern identification tool or clinical Korean medicine doctors' diagnosis of cold pattern. In addition, information regarding the levels of thyroid or adrenal hormones of the subjects is missing, even though these levels are presumed to be the biological basis of the cold patterns in this study.

Thus, future studies need a larger number of subjects of various ages and from different ethnic groups to confirm the generalization of our findings. In addition, for clinical application, large clinical studies are needed to determine whether the results of this study can be used in disease-based pattern identification. Additionally, it would be useful to study the association between the cold pattern and facial morphological features defined in a 3D space.

\section{Conclusions}

In this study, correlations between cold pattern and facial morphological features were analyzed with a fully automated facial shape analyzing system. The subjects with high CPS had a more pointed chin, a longer facial shape, more angular jaw, higher eyes, more upturned corners of the mouth, and relatively more widespread sensory organs of the face. These results suggest that facial diagnosis can be performed objectively for cold pattern identification. To the best of our knowledge, this study is the first to investigate the correlation between facial shape and cold pattern and suggests that facial morphology analysis is useful for identifying cold pattern.

\section{Acknowledgements}

This work was supported by the "Development of Korean Medicine Original Technology for Preventive Treatment Based on Integrative Big 
Data" grant from the Korea Institute of Oriental Medicine (KSN2022120).

\section{References}

1. Ferreira AS. \& Lopes AJ. (2011). Chinese medicine pattern differentiation and its implications for clinical practice. Chin $J$ Integr Med. 17(11), 818-823. doi: 10.1007/ s11655-011-0892-y

2. Choi, Y., Kim, S.D., Kwon, O., Park, H.J., Kim, J., Choi, W., Ko, M.H., Ha, S.J., Song, S.Y., Park, S.J. \& Yoo, H.S., (2021). Cold-Heat and Excess-Deficiency Pattern Identification Based on Questionnaire, Pulse, and Tongue in Cancer Patients: A Feasibility Study. Journal of Korean Medicine, 42(1), 1-11. doi: https://doi.org/10.13048/jkm.21001

3. Ko M, Lee J, Yun K, You S, and Lee M. Perception of pattern identification in traditional medicine: A survey of Korean medical practitioners. (2014). J Tradit Chin Med. 34(3), 369-372. doi: 10.1016/s0254 $-6272(14) 60104-7$

4. Zhu B \& Wang H. (2011). Diagnostics of Traditional Chinese Medicine. Singing Dragon.

5. Mun S, Kim S, Bae KH, \& Lee S. (2017). Cold and Spleen-Qi Deficiency Patterns in Korean Medicine Are Associated with Low Resting Metabolic Rate. Evidence-based Complement Altern Med. doi:10.1155/2017/ 9532073

6. Pham DD, Lee J, Kim G, Song J, Kim J, \& Leem CH. (2016). Relationship of the Cold-Heat Sensation of the Limbs and Abdomen with Physiological Biomarkers.
Evidence-based Complement Altern Med. doi:10.1155/2016/2718051

7. Park YJ, Lee JM, \& Park YB. (2013). Relationships between oriental medical pattern diagnosis and cardiovascular autonomic function. Eur J Integr Med. 5(6), 506-513. doi:10.1016/ j.eujim.2013.07.007

8. Lee M, Choi Y, Oriental G, \& Hospital M. (2015). Systemic reviews of domestic experimental studies of herbal medicines used for hypothyroidism since 2000. J Int Korean Med. 36(4), 570-581.

9. Ma T, Tan C, Zhang H, Wang M, Ding W, \& Li S. (2010). Bridging the gap between traditional Chinese medicine and systems biology: The connection of Cold Syndrome and NEI network. Mol Biosyst. 6(4), 613-619. doi:10.1039/b914024g

10. Henderson KE, Baranski TJ, Bickel PE, \& Clutter WE. (2008). The Washington Manual Endocrinology Subspecialty Consult. Vol 174. Lippincott Williams \& Wilkins.

11. Prout L. (2000). Live in the Balance: The Ground-Breaking East-West Nutrition Program. Da Capo Press.

12. Li X, Li F, Wang Y, Qian P, \& Zheng X. (2009). Computer-aided disease diagnosis system in TCM based on facial image analysis. Int $J$ Funct Inform Personal Med. 2(3), 303-314. doi:10.1504/IJFIPM.2009.030829

13. Liu M \& Guo Z. (2008). Hepatitis diagnosis using facial color image. International Conference on Medical Biometrics. Springer; 160-167. doi: 10.1007/978-3-540-77413-6_21

14. CAO Y, LI F, WANG Y, ZHENG X, DONG M, YAN H, \& QIAN P. (2013). Analysis on 
Facial Color Diagnosis for CHD Patients. Chinese Archives of Traditional Chinese Medicine, 31(9), 0-2. doi:10.13193/j.issn. 1673-7717.2013. 09.103

15. Zhang B, Vijaya Kumar BVK, \& Zhang D. (2014). Noninvasive diabetes mellitus detection using facial block color with a sparse representation classifier. IEEE Trans Biomed Eng. 61(4), 1027-1033. doi:10.1109/TBME. 2013.2292936.

16. Windhager S, Bookstein FL, Millesi E, Wallner B, \& Schaefer K. (2017). Patterns of correlation of facial shape with physiological measurements are more integrated than patterns of correlation with ratings. Scientific reports. 7(1), 1-11.

17. Henderson AJ, Holzleitner IJ, Talamas SN, \& Perrett DI. (2016). Perception of health from facial cues. Phil Trans $R$ Soc $B$. 371(1693), 20150380. doi: 10.1098/rstb.2015. 0380

18. Kocabey E, Camurcu M, Ofli F, Aytar Y, Marin J, Torralba A, \& Weber I. (2017). Face-to-BMI: Using computer vision to infer body mass index on social media. InProceedings of the International AAAI Conference on Web and Social Media 2017 May 3 (Vol. 11, No. 1).

19. Tai CH \& Lin DT. (2015). A Framework for Healthcare Everywhere: BMI Prediction Using Kinect and Data Mining Techniques on Mobiles. Proc - IEEE Int Conf Mob Data Manag. 2, 126-129. doi:10.1109/MDM.2015.40

20. Lee BJ \& Kim JY. (2014). Predicting visceral obesity based on facial characteristics. BMC Complement Altern Med. 14(1), 248. doi:10.1186/1472 -6882-14-248

21. Weber I \& Mejova Y. (2016). Crowdsourcing Health Labels: Inferring Body Weight from Profile Pictures. Proc 6th Int Conf Digit Heal Conf. 105-109. doi:10.1145/2896338.2897727

22. Nam J, Jang J-S, Kim H, Kim JY, \& Do J-H. (2017). Modification of the Integrated Sasang Constitutional Diagnostic Model. Evidence-Based Complement Altern Med. doi: https://doi.org/10.1155/2017/9180159

23. Koo I, Kim JY, Kim MG, \& Kim KH. (2009). Feature selection from a facial image for distinction of sasang constitution. Evidence-Based Complement Altern Med. 6(S1), 65-71. doi: https://doi.org/10.1093/ ecam/nep065

24. Kondo M, Okamura Y. (1987). Cold constitution: Analysis of the questionnaire. Nihon Sanka Fujinka Gakkai Zasshi. 39(11), 2000-2004.

25. Sadakata M, Yamada Y. (2007). Perception of foot temperature in young women with cold constitution: Analysis of skin temperature and warm and cold sensation thresholds. $J$ Physiol Anthropol. 26(4), 449-457. doi: 10.2114/jpa2.26.449.

26. Hur Y-M, Yu H, Jin H-J, \& Lee S. (2018). Heritability of cold and heat patterns: A twin study. Twin Res Hum Genet. 21(3), 227-232. doi: 10.1017/thg.2018.17

27. Wang Q, Yao S. (2008). Molecular basis for cold-intolerant yang-deficient constitution of traditional Chinese medicine. Am J Chin Med. 36(05), 827-834. doi: 10.1142/S0192415 X08006272.

28. Jin H-J, Baek Y, Kim H-S, Ryu J, \& Lee S. (2015). Constitutional multicenter bank linked 
to Sasang constitutional phenotypic data. BMC Complement Altern Med. 15, 46. doi:10.1186/s12906-015-0553-3

29. King DE. (2009). Dlib-ml: A Machine Learning Toolkit. J Mach Learn Res. 10, 1755-1758. doi:10.1145/1577069.1755843

30. Kazemi V, Sullivan J. (2014). One millisecond face alignment with an ensemble of regression trees. In Proceedings of the IEEE conference on computer vision and pattern recognition (1867-1874). doi:10.13140/2.1. 1212.2243

31. Gross R, Matthews I, Cohn J, Kanade T, \& Baker S. (2010). Multi-PIE. Image Vis Comput. 28(5), 807-813. doi:10.1016/j.imavis. 2009.08.002

32. Bae KH, Yoon Y, Yeo M, Kim HS, Lee Y, \& Lee S. (2016). Development on the questionnaire of cold-heat pattern identification based on usual symptoms for health promotion. $J$ Soc Prev Korean Med. 20, 17-26.

33. Seo JH, Park YB, \& Park YJ. (2014). Reliable facial color analysis using a digital camera and its relationship with pathological patterns: A pilot study. Eur J Integr Med. 6(3), 322-327. doi:10.1016/j.eujim.2014.02.002

34. Mun S, Ahn I, \& Lee S. (2017). The Association of Quantitative Facial Color Features with Cold Pattern in Traditional East Asian Medicine. Evidence-Based Complement Altern Med. doi: https://doi.org/10.1155/2017/ 9284856

35. Lu C, Xiao C, Chen G, Jiang M, Zha Q, Yan X, Kong W, \& Lu A. (2012). Cold and heat pattern of rheumatoid arthritis in traditional Chinese medicine: Distinct molecular signatures indentified by microarray expression profiles in CD4-positive T cell. Rheumatol Int. 32(1), 61-68. doi:10.1007/s00296-010-1546-7

36. Lee BJ, Lee JC, Nam J, \& Kim JY. (2016). Prediction of cold and heat patterns using anthropometric measures based on machine learning. Chin J Integr Med. 1-8. doi:10.1007/ s11655-016 -2641-8

37. Park AY, Cha S. (2017). Effects of cold sensitivity in the extremities on circulating adiponectin levels and metabolic syndrome in women. BMC Complement Altern Med. 17(1), 150. doi:10.1186/s12906-017-1658-7

38. Choi, Y., Kim, S.D., Kwon, O., Park, H.J., Kim, J., Choi, W., Ko, M.H., Ha, S.J., Song, S.Y., Park, S.J. \& Yoo, H.S., (2021). Cold-Heat and Excess-Deficiency Pattern Identification Based on Questionnaire, Pulse, and Tongue in Cancer Patients: A Feasibility Study. Journal of Korean Medicine, 42(1), 1-11. doi: https://doi.org/10.13048/jkm.21001

39. Storring M. (2004). Computer vision and Human Skin color. Faculty of Engineering and Sciencem Aalborg University, Niels Jernes Vej. 14, 9220.

40. Bae KH, Jang ES, Park K, \& Lee Y. (2018). Development on the questionnaire of cold-heat pattern identification based on usual symptoms: Reliability and validation study. Journal of physiology \& pathology in Korean Medicine. 32(5), 341-6. doi: https://doi.org/10.15188/ kjopp.2018.10.32.5.341 


\section{ORCID}

Ilkoo Ahn https://orcid.org/0000-0002-2074-0781

Kwang-Ho Bae https://orcid.org/0000-0001-7759-4650

Hee-Jeong Jin https://orcid.org/0000-0002-4373-3410

Siwoo Lee https://orcid.org/0000-0003-2658-8175 
The supplementary material of 'The Association between Facial Morphology and Cold Pattern'. The inclusion and exclusion criteria for participants

Inclusion Criteria Inclusion Criteria

1. Men and women aged 30 to 50 years

2. People who complain about sleeping problems more than 3 times a week (when it takes more than 30 minutes to get in or when it takes more than 30 minutes to re-enter after awakening, or when it is difficult to maintain sleep)

3. Those with an Insomnia Severity Index (ISI) of 8 or higher and 21 or lower

4. Mibyeong survey score of 14 points or more

\section{Exclusion Criteria}

1. Those who have received medical or Korean medical treatment for sleep problems within the last 1 month

2. Those who are taking medication or receiving treatment within the last 1 month due to nervous system and psychiatric disorders (depression, anxiety disorder, dementia, etc.)

3. Those who have been diagnosed with a disease that may cause other insomnia (sleep apnea, restless legs syndrome, narcolepsy, etc.)

4. Those who have abused or been dependent on alcohol within the last 6 months

5. Those who are working at night, on shift, or in heavy redundancy

6. People who have no problems with sleep items of Mibyeong survey

7. Pregnant women, women who are breastfeeding and who have a pregnancy plan within 6 months

8. Those who believe that they cannot take medications that may affect clinical studies or that they cannot comply with other test compliance 